\title{
Equivalent Acceleration Assessment of JEDEC Moisture Sensitivity Levels using Peridynamics
}

\author{
Sungwon Han, Seyoung Lim, Jangyoung Bae, Yuchul Hwang, Sungsoo Lee \\ Samsung Electronics Co., LTC, Korea \\ Selda Oterkus, and Erdogan Madenci \\ University of Arizona, Tucson, AZ \\ Cagan Diyaroglu and Erkan Oterkus \\ University of Stratchclyde, Glasgow, UK
}

\begin{abstract}
The moisture inside the IC packages induces the several deformation failures, such as popcorn crack and swelling during the solder reflowing process. In semiconductor industry, over the past few years, the equivalent acceleration time for JEDEC moisture sensitivity level has been updated based on the weight gain measurements when the package structure and materials were modified. It costs long test times which may induce the significant delay of new product development and reliability evaluation. Additionally, the weight gain equivalency may not be sufficient to determine the equivalent accelerated time. In this paper, the new approach for evaluating the equivalent acceleration test time for preconditioning is proposed using the numerical calculation by peridynamics (PD) theory. The essential of proposed method is analyzing a moisture concentration and a vapor pressure which can cause the moisture induced failure in IC packages without facing the discontinuity problems of moisture concentration along the interfaces.
\end{abstract}

\section{Introduction}

Integrated circuit (IC) packages the have likelihood to absorb the moisture between the polymeric and nonpolymeric materials during their packaging, transportation and storage. The moisture inside the packages causes cracking and delamination failures during soldering reflowing process due to the sudden vaporization of the absorbed moisture [1]. JEDEC standard (J-STD-020) defined and outlined the moisture absorption test conditions for plastic surface mount devices (SMDs) to classify the moisture sensitivity rating of plastic packages [2]. For example, the moisture sensitivity level 3, which is most commonly applied in our plastic package products, requires 192 hours moisture preconditioning at $30{ }^{\circ} \mathrm{C} / 60 \% \mathrm{RH}$. As accelerated equivalent test conditions for the moisture sensitivity level 3, 40 or 52 hours at $60^{\circ} \mathrm{C} / 60 \% \mathrm{RH}$ is recommended in JDEDC standard. However, in semiconductor industry, such long test times are impractical and induce the significant delay of new product development and reliability evaluation.

Traditionally, the weight gain measurements have been used to establish accelerated equivalent conditions for the moisture preconditioning because of its convenience. Today, semiconductor industries provide many different types of IC packages with several different structures and materials that can affect the moisture absorption characteristics. It implies the accelerated equivalent test conditions required to be updated more frequently than ever. However, the weight gain measurements require the long test times (192 or 168 hours) and it also has been reported that the comparison of the weight gain of IC packages may not correlate with a moisture concentration inside the IC packages [3-5]. Kitano et al. demonstrated the package cracking is not controlled by the absolute weight gain of packages, but the local moisture concentration at the critical interface [3]. It means that only considering the percent weight gain equivalency is not sufficient to establish the equivalent accelerated time, because the moisture induced failure is due to the combination of thermo-mechanical, hygroscopic stress and, and vapor pressure. At the reflow temperature, the vapor pressure strain is important as thermo-mechanical and hygro-mechanical strains since the modulus of polymer materials drops a few orders [3]. Therefore, the weight gain measurement may not provide the enough information in terms of moisture induced failure. It is the reason why the moisture diffusion and vapor pressure analysis inside the IC packages should be considered as the key point to figure out the moisture induced failure in electronic packages.

Currently, there is no known technique for measurement of moisture concentration distribution inside the IC packages, so the numerical calculation based on the finite element method (FEM) was used to analyze the moisture diffusion inside the IC packages. In FEM, the moisture diffusion analysis is performed by using the thermal-moisture analogy because the moisture diffusion behavior can be described using the Fick's law. However, the moisture concentration is usually discontinuous along bi-material interfaces because the saturated concentration of each material is not identical [6-9]. In order to overcome the moisture concentration discontinuous at interfaces, Wong et al. introduced the "wetness", which is the normalized variable defined as the ratio of moisture concentration over its saturated moisture concentration [7]. Also, Fan et al. proposed a direct concentration approach (DCA) by imposing the continuity condition along the interface between dissimilar materials because the saturation moisture concentration is not constant during the reflow process [8-10]. Madenci et al. proposed the integrated approach for the simulation of hygro-thermo-vapordeformation analysis using peridynamic theory that permits the direct modeling of moisture diffusion $[11,12]$

In this paper, the new approach for determining the equivalent acceleration test condition of JEDEC moisture sensitivity level considering the moisture diffusion and vapor 
pressure inside the package is proposed. In numerical calculation, the peridynamic theory which is a nonlocal continuum theory is employed to overcome the difficulties arising due to the existence of discontinues between bimaterial interfaces [11-14]. The detailed information regarding peridynamic is described in next section.

\section{Experiments and Numerical Calculations}

\section{- Experiments}

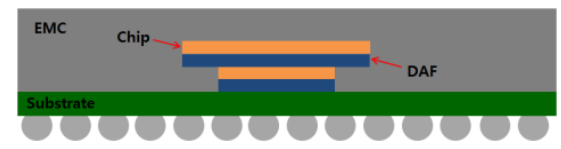

Fig. 1. The schematic of dual die package for LPDDR3

Moisture absorption experiments were performed using a 220 pin dual die packages (DDP: $14 \times 14 \times 0.85 \mathrm{~mm}$ ) for DRAM product (LPDDR3). The schematic of test IC package is described in Fig. 1. The moisture sensitivity level 2 was selected for absorption test conditions because it's the harsh conditions which may induced the moisture related failure and the accelerated equivalent conditions was not defined in JEDEC standard as well.

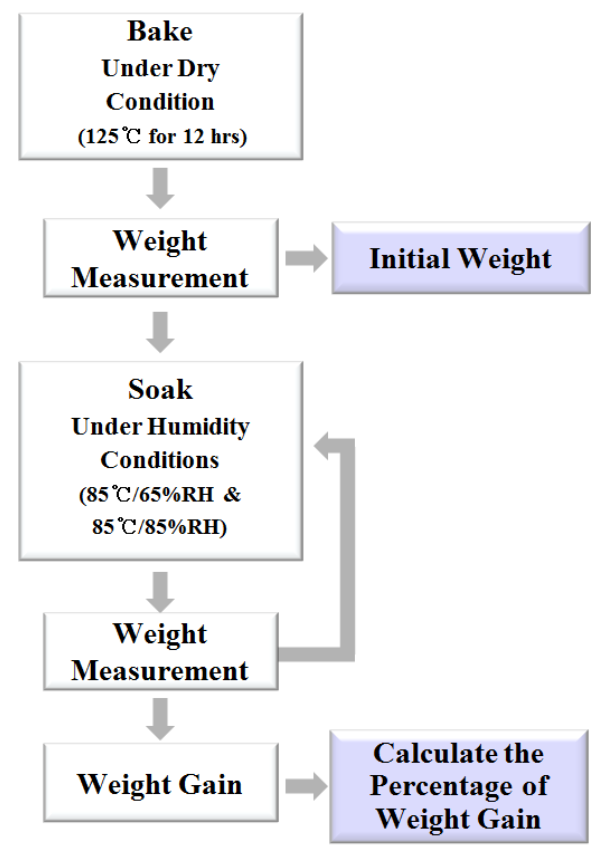

Fig. 2. Procedure for the weight gain measurement

The experimental procedure followed the flow as outlined in JEDEC standard (J-STD-020) [2]. Total 60 DDP packages were baked at $125^{\circ} \mathrm{C}$ for 12 hours to remove moisture from the package and the weight was measured as an initial weight at dry condition. It is recommended for 24 hours of baking process in JDEDC standard, 12 hours of baking time was applied because it was confirmed that test specimens showed no significant changes of weight loss after 12 hours of baking time. Then, "dry" packages were divided into two groups with 30 test specimens and were exposure under two different conditions of temperature and relative humidity $\left(85^{\circ} \mathrm{C} / 65 \% \mathrm{RH}\right.$ and $\left.85^{\circ} \mathrm{C} / 85 \% \mathrm{RH}\right)$. The increase of moisture weight gain inside the specimen was measured as a function of time until 192 hours at $85^{\circ} \mathrm{C} / 65 \% \mathrm{RH}$ and 69 hours at $85^{\circ} \mathrm{C} / 85 \% \mathrm{RH}$ conditions in order to calculate the percentage of weight gain. When the test specimens were removed from the environmental chamber to measure the weight gain, the measurement was not exceeding more than 5 minutes to minimize the weight loss during the measurement which may affect the accuracy of weight gains. The procedure for weight gain measurement is depicted in Fig. 2.

\section{- Basics of Peridynamics}

The peridynamic theory uses spatial integral equations rather than the partial differential equations used in the classical continuum mechanics which was introduced by Silling [11-14]. The governing equations of peridynamic and classical continuum mechanics as given in the following equations:

$$
\begin{aligned}
& \text { Peridynamics: } \rho \ddot{u}=\int \mathrm{fdV}+b \quad \text { (1) } \\
& \text { Classical continuum mechanics: } \rho \ddot{u}=\nabla \sigma+b
\end{aligned}
$$

where $\rho$ is a mass density, $\ddot{u}$ is an acceleration, $\mathrm{f}$ is a bond force which is a function of displacement and $b$ is body force.

In classical continuum mechanics, the divergence of stress in Eq. (1) requires the displacement fields should be continuous. However, in peridynamics, each point is connected to all points within a region of finite radius (length parameter called as "horizon") by bond force. The interaction of material points can be described as a response function which contains all constitutive information associated with the materials [11,12]. Depending on the horizon value, the locality of interactions can become more local when the horizon decreases as shown in Fig. 3.

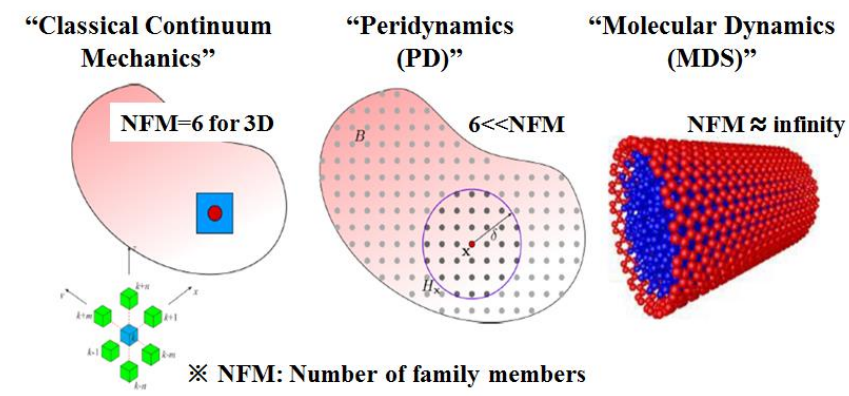

Fig. 3. Comparison between local and nonlocal model [13] 
Table I Material properties at $85^{\circ} \mathrm{C} / 85 \% \mathrm{RH}$ and $85^{\circ} \mathrm{C} / 65 \% \mathrm{RH}$ conditions

\begin{tabular}{cccc}
\hline \hline & EMC & DAF & PCB \\
\hline $\begin{array}{c}\text { Moisture diffusivity D }\left(\mathbf{m}^{2} / \mathbf{h r}\right) @ \mathbf{8 5}^{\circ} \mathrm{C} \\
\text { Saturated moisture concentration } \\
\quad \mathbf{C}_{\text {sat }}\left(\mathbf{k g} / \mathbf{m}^{3}\right) @ \mathbf{8 5}^{\circ} \mathbf{C} / \mathbf{8 5 \%} \mathbf{R H}\end{array}$ & $6.2 \times 10^{-9}$ & $2792 \times 10^{-9}$ & $582 \times 10^{-9}$ \\
$\begin{array}{c}\text { Saturated moisture concentration } \\
\mathbf{C}_{\text {sat }}\left(\mathbf{k g} / \mathbf{m}^{\mathbf{3}}\right) @ \mathbf{8 5}{ }^{\circ} \mathbf{C} / \mathbf{6 5 \%} \mathbf{R H}\end{array}$ & 6.1 & 20.8 & 4.4 \\
\hline \hline
\end{tabular}

Peridynamic theory is very suitable for modeling bodies with evolving discontinuities such as cracks and discontinuous issues in moisture concentration. Especially, in peridynamics, any additional damage theory such as fracture mechanics is not needed because it is essentially based on the bond strength between points as described in Eq. (2). Therefore, the material damage which can represent the crack initiation or propagation can be determined when the interactions among the material points were eliminated. In peridynamic theory, it is assumed that the damage occurs when the stretching between material points exceeds a critical value that called as critical stretch [11-14]. Peridynamic offers a unique capability for analyzing the moisture concentration by overcoming the discontinuity along the bi-material interface and it can also effectively predict the damage initiation and growth due to the presence of temperature and moisture during the reflow process.

\section{- Numerical Calculations}

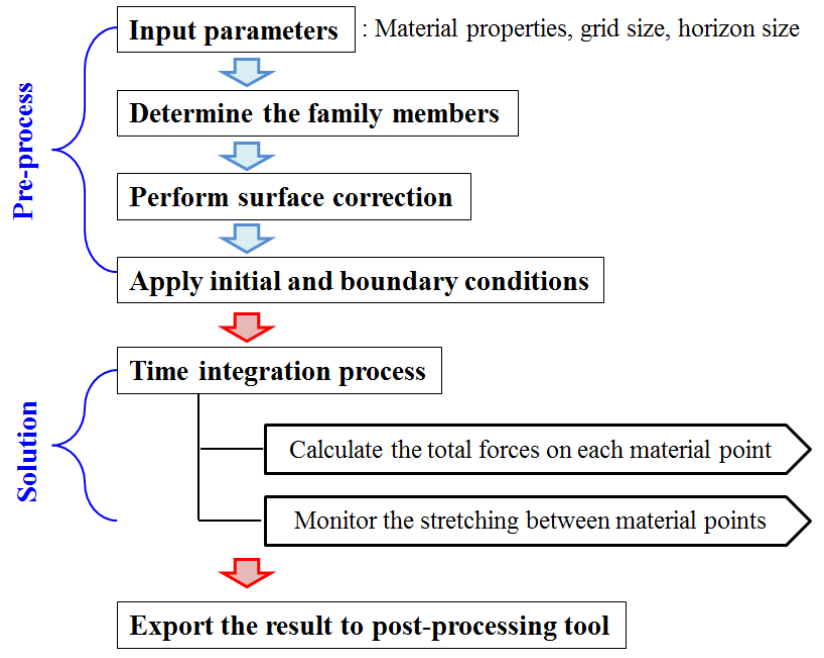

Fig. 4. Flowchart of peridynamic simulation process

The simulation process by using a peridynamic theory is depicted in Fig. 4. Compare to the widely used computational tool, finite element method (FEM), the peridynamic theory based simulation does not including the mesh process because peridynamics is the nonlocal model. Peridynamics normally discretize the domain into small volumes and represent each volume with a node (material point). The programming language being used is FORTRAN and the contours of both moisture distribution and vapor pressure distribution are graphically visualized using MATLAB.

The material properties, i.e. diffusivity (D) and saturated moisture concentration (Csat) at $85^{\circ} \mathrm{C} / 85 \% \mathrm{RH}$ at $85^{\circ} \mathrm{C} / 65 \% \mathrm{RH}$ conditions are listed in Table 1.

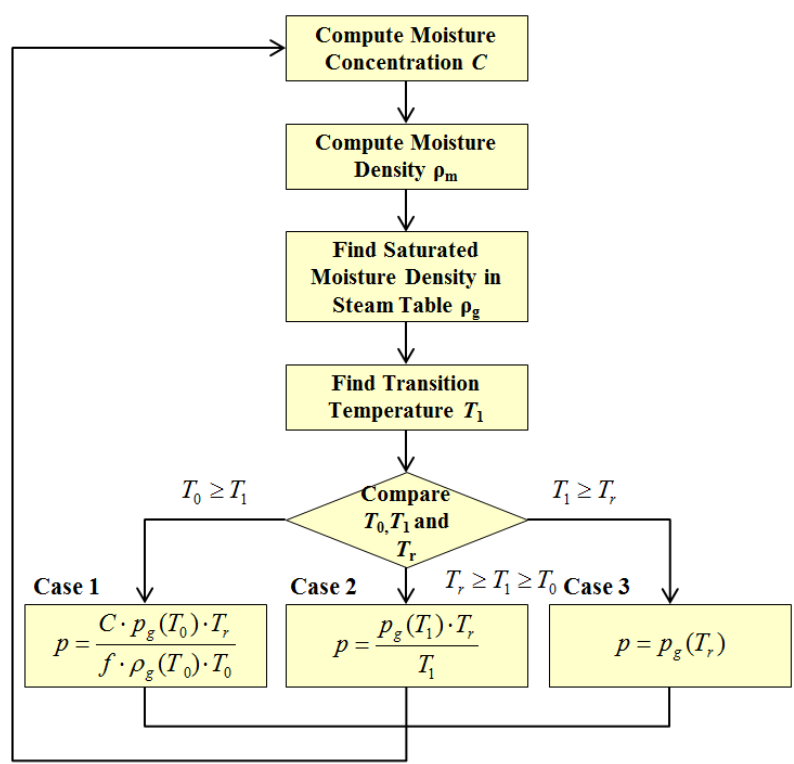

Fig. 5. Flowchart of vapor pressure simulation

In general, the moisture induced failures are observed after the reflow process due to the vaporization of moisture absorbed inside the IC packages. It implies that the strength of internal vapor pressure generated in reflow process is critical to determine the likelihood of failures due to the moisture absorption. To analyze the vapor pressure inside the IC packages, the micromechanics-based vapor pressure model introduced by Fan is used [9]. Fan described the moisture states in the pores in three distinct cases assumed that the voids inside polymer are uniformly distributed $[8,9]$. The vapor pressure (p) at each node can be calculated by flowchart depicted in Fig. 4.

Case 1: All the moisture becomes vaporized at preconditioning temperature $\left(\mathrm{T}_{0}\right)$ due to the moisture density in the voids is low.

Case 2: Moisture is not fully vaporized even at reflow temperature $\left(T_{r}\right)$. 


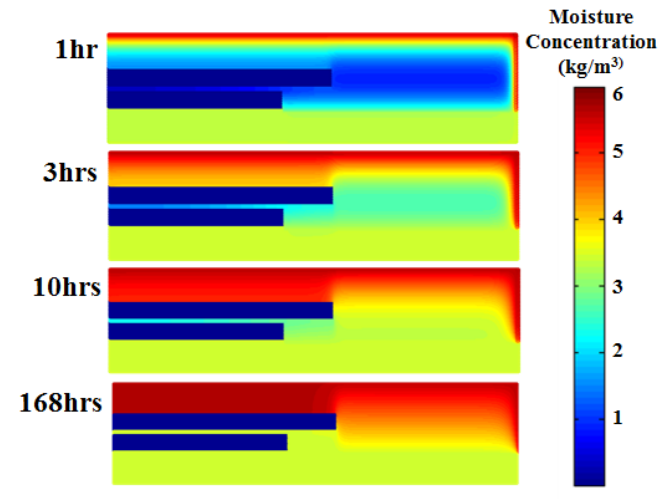

Fig. 7 Overall moisture concentration distribution at $85^{\circ} \mathrm{C} / 65 \% \mathrm{RH}$ conditions

Case 3: Moisture is fully vaporized at Tr.

In which, $T_{r}$ is the transition temperature, $\mathrm{p}_{\mathrm{g}}(\mathrm{T})$ is the saturated pressure, $\rho_{\mathrm{g}}(\mathrm{T})$ is the saturated moisture density, and $f$ is the void volume fraction with its initial value being $f_{0}$. The initial volume fraction $\mathrm{f}_{0}$ is assumed to be 0.05 for EMC and DAF. It is reported that the effect of $f_{0}$ on vapor pressure is not critical [6]. It may be related the adhesion of interfaces but the effect of interface adhesion is not considered in this study.

\section{Results and Discussion}

\section{- Weight Gain Measurement}

The weight gain measurements at $85^{\circ} \mathrm{C} / 85 \% \mathrm{RH}$ and $85^{\circ} \mathrm{C} / 65 \% \mathrm{RH}$ conditions showed in Fig. 6 . The weight gains of the specimens were saturated over time. The moisture absorption of package shows a dual-stage non-Fickian behavior. The first mechanism is the absorption of water molecules in the free volumes or nano-voids. Other is the hydrogen bonding between the water molecules and polymer chains due to their molecular polarity [15].

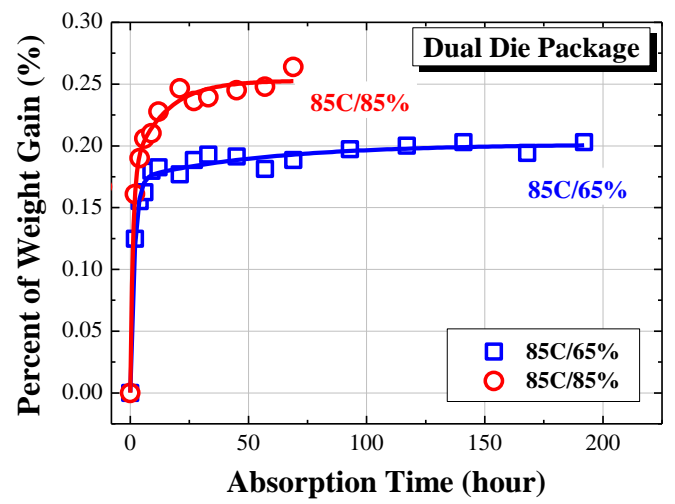

Fig. 6. The percentage of weight gains from $85^{\circ} \mathrm{C} / 65 \% \mathrm{RH}$ and $85^{\circ} \mathrm{C} / 85 \% \mathrm{RH}$ conditions

The accelerated equivalent test time in $85^{\circ} \mathrm{C} / 85 \% \mathrm{RH}$ conditions can be calculated based on the time which can obtain the same percent of weight gain from 168 hours in $85^{\circ} \mathrm{C} / 65 \% \mathrm{RH}$ conditions. In our absorption test, the percent of

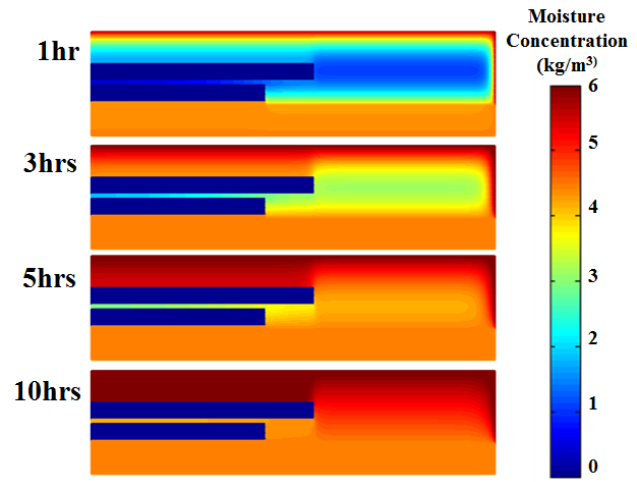

Fig. 8 Overall moisture concentration distribution at $85^{\circ} \mathrm{C} / 85 \% \mathrm{RH}$ conditions

weight gain after 168 hours at $85^{\circ} \mathrm{C} / 65 \% \mathrm{RH}$ conditions is approximately $0.2 \%$. The same percent of weight gain can be measured after approximately 5 hours at $85^{\circ} \mathrm{C} / 85 \% \mathrm{RH}$ conditions. It implies that amount of moisture inside the packages at $85^{\circ} \mathrm{C} / 85 \% \mathrm{RH}$ for 5 hours is approximately identical compared to the moistures absorption after the 168 hours exposure at $85^{\circ} \mathrm{C} / 65 \% \mathrm{RH}$ conditions. Therefore, 5 hours at $85^{\circ} \mathrm{C} / 85 \% \mathrm{RH}$ conditions can be used as the accelerated equivalent time for moisture sensitivity level 2 based on the weight gain measurement.

\section{- Moisture Concentration Distribution}

The overall moisture distribution inside the package after the 1,10 and 168 hours of absorption at $85^{\circ} \mathrm{C} / 65 \% \mathrm{RH}$ conditions which is analyzed using peridynamic simulation showed in Fig. 7. The PCB and 1st DAF were fully saturated after 1 hour of absorption, while the moisture was gradually diffused into the EMC area, because the high diffusivity of PCB $\left(582 \mathrm{~m}^{2} / \mathrm{hr}\right)$ compared to that of EMC $\left(6.2 \mathrm{~m}^{2} / \mathrm{hr}\right)$. As increasing the absorption time, the moisture concentration at 2nd DAF is increased by the gradually diffused moisture sources from the PCB and EMC. After 168 hours, the moisture concentration at 2nd DAF was equalized as approximately $3.5 \mathrm{~kg} / \mathrm{m}^{3}$

Fig. 8 shows the overall moisture distribution under the $85^{\circ} \mathrm{C} / 85 \% \mathrm{RH}$ conditions. The peridynamic simulation result shows the moisture concentration inside the package and the moisture distribution is identical between that of $85^{\circ} \mathrm{C} / 85 \% \mathrm{RH}$ for 5 hours and $85^{\circ} \mathrm{C} / 65 \%$ for 168 hours, while the accelerated equivalent time based on percent of weight gain was 5 hours at $85^{\circ} \mathrm{C} / 85 \% \mathrm{RH}$. The moisture couldn't diffuse into the left side $2^{\text {nd }}$ DAF which is the center of package compared to that from $85^{\circ} \mathrm{C} / 65 \%$ for 168 hours, so more absorption time requires to become the moisture concentration equivalency.

The local moisture concentration at the center of package where the center of $2^{\text {nd }}$ DAF was chose to determine the accelerated equivalent test time as shown in Fig. 9. As shown in Fig. 7 and 8, the moisture concentration was saturated after 20 hours both at $85^{\circ} \mathrm{C} / 85 \% \mathrm{RH}$ and $85^{\circ} \mathrm{C} / 65 \% \mathrm{RH}$ conditions. Considering the local moisture concentration, 6.5 hours at 
$85^{\circ} \mathrm{C} / 85 \% \mathrm{RH}$ conditions can be the equivalent test condition for the moisture sensitivity level 2 .

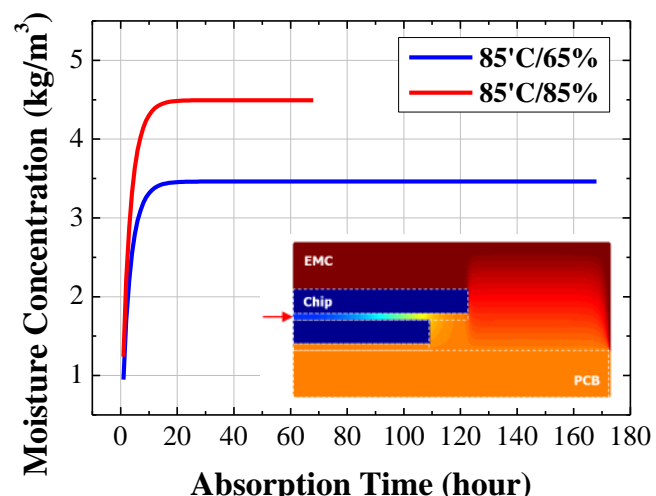

Fig. 9 Change of local moisture concentration at 2nd DAF and chip interface (Center of DDP package)

\section{- Vapor Pressure Distribution}

The vapor pressure distributions of DDP package under $85^{\circ} \mathrm{C} / 65 \% \mathrm{RH}$ and $85^{\circ} \mathrm{C} / 85 \% \mathrm{RH}$ conditions depending on the absorption time are plotted in Fig. 10.

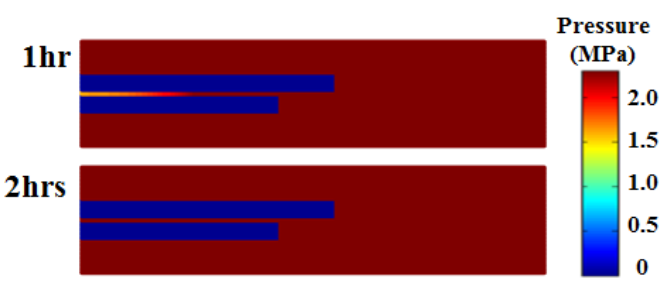

(a) $85^{\circ} \mathrm{C} / 65 \% \mathrm{RH}$ conditions

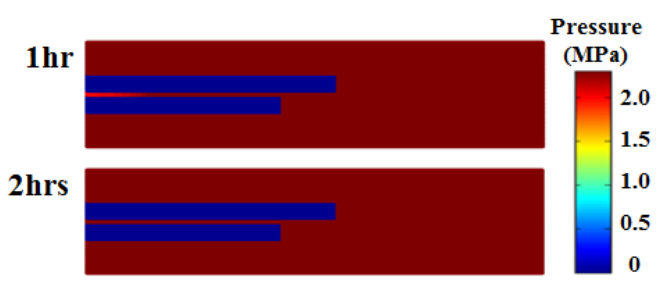

(b) $85^{\circ} \mathrm{C} / 85 \% \mathrm{RH}$ conditions

Fig. 10 Vapor pressure distribution under $85^{\circ} \mathrm{C} / 65 \% \mathrm{RH}$, and (b) Under $85^{\circ} \mathrm{C} / 85 \% \mathrm{RH}$ conditions

The vapor pressure at $2 \mathrm{nd} \mathrm{DAF}$ and chip interface reached saturated pressure (about $2.3 \mathrm{MPa}$ ) at reflow temperature after 2 hours both at $85^{\circ} \mathrm{C} / 85 \% \mathrm{RH}$ and $85^{\circ} \mathrm{C} / 65 \% \mathrm{RH}$ conditions when the package is far from the saturated moisture diffusion state at Fig. 6. The vapor pressure at 6.5 hours under $85^{\circ} \mathrm{C} / 85 \% \mathrm{RH}$ conditions which is the equivalent acceleration test for the moisture sensitivity level 2 based on the weight gain measurements showed the saturated pressure at the center of package as well.

The estimated equivalent accelerated time through the assessment methods are summarized in Table II.
Table II Equivalent accelerated time of JEDEC moisture sensitivity level 2 for DDP packages

\begin{tabular}{cc}
\hline \hline Method & $\begin{array}{c}\text { Equivalent } \\
\text { accelerated time at } \\
\mathbf{8 5}^{\circ} \mathbf{C} / \mathbf{8 5} \% \mathbf{R H}\end{array}$ \\
\hline Weight gain & 5 hours \\
Moisture concentration & 6.5 hours \\
Vapor pressure & 2 hours \\
\hline \hline
\end{tabular}

Compared to the moisture concentration distribution, the vapor pressure distribution is quite different. The vapor pressure in the package reaches the saturated pressure much faster than the saturated moisture concentration in moisture diffusion. It implies that the additional moisture absorption due to the longer absorption time does not increase the level of vapor pressure inside the package. In terms of vapor pressure inside the package, the equivalent accelerated time for JEDEC moisture sensitivity level 2 can be determined as approximately 2 hours at $85^{\circ} \mathrm{C} / 85 \% \mathrm{RH}$ conditions

\section{Conclusions}

This paper proposed the new approach for determining the equivalent acceleration conditions for JEDEC moisture sensitivity level based on the moisture diffusion and vapor pressure. In order to overcome the discontinuity of moisture concentration at bi-material material interfaces, the peridynamic (PD) theory was employed in the analysis of moisture diffusion and vapor pressure inside the package. The numerical calculation result by peridynamic theory shows that weight gain measurements may not correlate with the moisture concentration at critical interface (center of IC package). Peridynamic simulation also demonstrates the vapor pressure saturated earlier than moisture diffusion, which implies that vapor pressure may remain the same pressure level regardless of the increment of moisture absorption duration.

\section{Acknowledgments}

The authors would like to acknowledge the work and discussion regarding the peridynamic theory and moisture diffusion and vapor pressure simulation code with Pro. Madenci and Dr. Oterkus from University of Arizona, and Dr. Oterkus from University of Strathclyde.

\section{References}

1. Fukuzawa, I. et al. Moisture Resistance Degradation of Plastic LSI's by Reflow Soldering. ECTC IRPS (1985)

2. J-STD-020D Moisture/reflow sensitivity classification for non-hermetic solid state surface mount devices (2008)

3. Kitano, M. et al. Analysis of Package Cracking During Reflow Soldering Process. IEEE IRPS (1988)

4. Richard, S. et al. Method for equivalent acceleration of JEDEC/IPC moisture sensitivity levels. IEEE IRPS (1998)

5. Shi, D. et al. A new method for equivalent acceleration of JEDEC moisture sensitivity levels, IEEE ECTC (2008) 
6. Tong, T. and Hun, Ng, Whole Field Vapor Pressure Modeling of QFN during Reflow with Coupled Hygromechanical and Thermo-mechanical stress. IEEE ECTC (2002)

7. Wong, E. et al. The Mechanics and Impact of Hygroscopic Swelling of Polymeric Materials in Electronic Packaging. IEEE ECTC (2000)

8. Fan, X. et al. Interfacial Delamination Mechanisms During Soldering Reflow with Moisture Preconditioning. IEEE Trans. Compon., Packag. (2008)

9. Fan, X. et al. A Micromechanics based Vapor pressure Model in Electronic Packages. ASME J. Electron. Packag. (2005)

10. Xie, B. et al. Direct Concentration Approach of Moisture Diffusion and Whole-Field Vapor Pressure Modeling for Review Process-Part I: Theory and Numerical Implementation. ASME J. Electron. Packag. (2009)

11. Madenci, E. and Oterkus, E. Peridynamic Theory and Its Applications. Springer (2013)

12. Oterkus, S. Hygro-Thermo-Mechanical Analysis and Failure Prediction in Electronic Packages by Using Peridynamics. IEEE ECTC (2014)

13. Silling, SA. Reformulation of elasticity theory for discontinuities and long-range forces. J Mech Phys Solids (2000)

14. Silling, SA. et al. Peridynamics states and constitutive modeling. J Elast (2007)

15. Shirangi, MH. Et al. Mechanism of Moisture Diffusion, Hygroscopic Swelling and Adhesion Degradation in Epoxy Molding Compounds. IMAPS (2008) 